\title{
Geometric phases for corotating elliptical vortex patches
}

\author{
B. N. Shashikanth ${ }^{\text {a) }}$ \\ Control and Dynamical Systems, California Institute of Technology, \\ Mail Code 107-81, Pasadena, California 91125 \\ P. K. Newton ${ }^{\text {b) }}$ \\ Department of Aerospace and Mechanical Engineering and Department of Mathematics, \\ University of Southern California, Los Angeles, California 90089-1191
}

(Received 16 December 1999; accepted for publication 6 September 2000)

We describe a geometric phase that arises when two elliptical vortex patches corotate. Using the Hamiltonian moment model of Melander, Zabusky, and Styczek [J. Fluid Mech. 167, 95-115 (1986)] we consider two corotating uniform elliptical patches evolving according to the second order truncated equations of the model. The phase is computed in the adiabatic setting of a slowly varying Hamiltonian as in the work of Hannay [J. Phys. A 18, 221-230 (1985)] and Berry [Proc. R. Soc. London, Ser. A 392, 45-57 (1984)]. We also discuss the geometry of the symplectic phase space of the model in the context of nonadiabatic phases. The adiabatic phase appears in the orientation angle of each patch-it is similiar in form and is calculated using a multiscale perturbation procedure as in the point vortex configuration of Newton [Physica D 79, 416-423 (1994)] and Shashikanth and Newton [J. Nonlinear Sci. 8, 183-214 (1998)], however, an extra factor due to the internal stucture of the patch is present. The final result depends on the initial orientation of the patches unlike the phases in the works of Hannay and Berry [J. Phys. A 18, 221-230 (1985)]; [Proc. R. Soc. London, Ser. A 392, 45-57 (1984)]. We then show that the adiabatic phase can be interpreted as the holonomy of a connection on the trivial principal fiber bundle $\pi: T^{2} \times S^{1} \rightarrow S^{1}$, where $T^{2}$ is identified with the product of the momentum level sets of two Kirchhoff vortex patches and $S^{1}$ is diffeomorphic to the momentum level set of two point vortex motion. This two point vortex motion is the motion that the patch centroids approach in the adiabatic limit. (C) 2000 American Institute of Physics. [S0022-2488(00)01312-8]

\section{INTRODUCTION}

A vortex patch is a desingularization of a point vortex in which the vorticity is a bounded function over a finite, nonzero area $A$ of the plane. It can thus be viewed as the perpendicular section of an infinitely long rectilinear vortex tube of area $A$ whose vorticity distribution is invariant along the length of the tube. The induced velocity field is given by integrating the contribution due to each infinitesimal vorticity element over the area of the patch. For a patch with vorticity distribution $\omega(x, y)$, the stream function is given by

$$
\psi(x, y)=-\frac{1}{4 \pi} \int_{A} \omega\left(x^{\prime}, y^{\prime}\right) \log \left[\left(x-x^{\prime}\right)^{2}+\left(y-y^{\prime}\right)^{2}\right] d A^{\prime}
$$

The velocity components $(u, v)$ at a point $(x, y)$ are related to the stream function in the usual fashion $u=\partial \psi / \partial y$ and $v=-\partial \psi / \partial x$.

A patch is uniform if $\omega(x, y)=$ constant. A simple example of an isolated uniform vortex patch, which is the basis for the model we consider, is the Kirchhoff elliptical vortex. ${ }^{1}$ Here the

\footnotetext{
${ }^{a)}$ Electronic mail: shashika@cds.caltech.edu

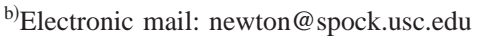


uniform vorticity is distributed over an elliptical region with aspect ratio $\lambda=a / b$ ( $a$ major axis, $b$ minor axis). The patch rotates about the center of the ellipse with constant angular velocity given by

$$
\Theta=\frac{\omega \lambda}{(1+\lambda)^{2}}=\frac{\Gamma \lambda}{A(1+\lambda)^{2}},
$$

where $\Gamma=\omega A$ is the patch strength. As shown in Lamb (section 159) a fluid particle on the patch moves in a circular orbit with twice this frequency. Deem and Zabusky ${ }^{2}$ have conjectured, based on numerical computations, that the Kirchhoff vortex is a member $(m=2)$ of a class of rotating patches (constant frequency) with $m$-fold symmetry. Overman and Zabusky ${ }^{3,4}$ have computed interactions of such $m$-fold "states." Kida ${ }^{5}$ showed that an elliptical vortex in a uniform shear flow maintains its elliptical shape. Nonlinear stability results for vortex patches can be found, among others, in Wan and Pulverenti, ${ }^{6}$ Wan, ${ }^{7}$ and Constantin and Titi. ${ }^{8}$

For a system of $N$ disjoint patches, the velocity field is obtained by linearly superposing the velocity field due to each patch. A system of $N$ interacting patches is in general far more complicated than a system of $N$ interacting point vortices due to the internal structure associated with each patch. There is a large body of numerical work documenting various complex processes associated with vortex patches, such as merger and filamentation. A good overview of work done on patches can be found in Chap. 9 of Saffman. ${ }^{9}$

It is well-known (see Refs. 10-13, and references therein) that the Euler equations, for which $N$ patch systems are solutions, posses a noncanonical Hamiltonian structure. The real-valued Hamiltonian is a functional of the vorticity field $\omega$ of the domain. The dynamics of the vorticity field can be expressed in the form,

$$
\frac{d F}{d t}=\{F, H\}
$$

where $F$ is any functional of $\omega$, and \{\} are appropriately defined Poisson brackets. This representation is equivalent to the dynamics represented by the Euler equations in the vorticity-streamfunction form.

Finite-dimensional Hamiltonian models for $N$ patch systems can be obtained using the moment model of Melander, Zabusky, and Styczek ${ }^{14,15}$ (henceforth referred to as the MZS model). In this paper we consider the evolution of two well-separated vortex patches in the plane as described by the MZS model. The problem we address is the calculation of a geometric phase in the second order truncated system of equations obtained from the model. Geometric phases, though having been known in other forms for more than a century, attracted serious attention with the work of Berry ${ }^{16}$ ("Berry's phase") and Hannay ${ }^{17}$ ("Hannay's angle") in the context of adiabatic evolutions of quantum and classical parameter-dependent Hamiltonians, respectively. The formal differential geometric interpretation of "Berry's phase" was first given by Simon. ${ }^{18}$ Later, Marsden, Montgomery, and Ratiu ${ }^{19}$ (see also Montgomery ${ }^{20}$ and an introduction in Marsden and Ratiu ${ }^{21}$ ) developed further the geometric theory in both adiabatic and nonadiabatic settings. We refer the reader to the references and discussion in Shashikanth and Newton ${ }^{22,23}$ and Newton ${ }^{24}$ for background as well as discussions of its relevance in the context of point vortex theory.

We show in this paper that geometric phases appear in the vortex patch motion modeled by the second order MZS system for two corotating (i.e., like-signed) patches. The second order MZS system models the patches as ellipses of varying eccentricities and nonuniform rates of rotation about their centroids which move approximately as point vortices. In other words the patches can be viewed as Kirchhoff ellipses with moving centroids and time-varying aspect ratios and angular velocities. The geometry of the symplectic phase space of the model is discussed first and some general calculations of the momentum map and the coadjoint isotropy subgroups are presented. We do not attempt to compute connections and phases on the Marsden-Weinstein bundles in this paper but we do make some general remarks in this context. Geometric phases are then calculated 
explicitly in an adiabatic setting defined as in the point vortex configurations considered in the work of Newton ${ }^{25}$ and Shashikanth and Newton. ${ }^{22,23,26}$ The two length scales in the problem, due to the interpatch separation and the patch size, define two corresponding time scales which represent the "slow" point-vortex-like motion of the centroids and the "fast" rotation rates of the Kirchhoff-ellipse-like patches, respectively. The question we address is: what is the change in the orientation angle of each patch at the end of one appropriately defined time period of the centroid motion? More specifically, can this angle change be expressed as the sum of a "dynamic" part and a "geometric" part? We show by a standard multiscale perturbation solution technique ${ }^{27}$ that this splitting is possible and interpret the "geometric" part as being a geometric phase arising as the holonomy of a connection defined on an appropriately defined fiber bundle. We also calculate phases in a four-point vortex configuration analogous to the patch problem where each patch is replaced by a like-signed point vortex pair of the same total strength as the patch.

The outline of the paper is as follows: in Sec. II we describe the MZS model for $N$ patches and its Hamiltonian structure. We show that, as in the planar $N$ point vortex problem, the momentum map for the $\operatorname{SE}(2)$ action is equivariant iff the sum of the patch strengths is zero. The isotropy subgroups for the coadjoint action in each case (equivariant and nonequivariant) are calculated, and we conclude by making some general remarks on connections and phases. In Sec. III we describe the adiabatic setting and give details of the multiscale asymptotic procedure for the calculation of the phase and the results. For comparision, we also present the results of an adiabatic phase calculation for a four point vortex problem analogous to the patch problem. The section concludes with the formal geometric interpretation of the adiabatic phase.

\section{HAMILTONIAN STRUCTURE OF THE MZS MODEL AND NONADIABATIC PHASES}

Melander, Zabusky, and Styczek ${ }^{14,15}$ derived a system of equations (henceforth referred to as the MZS model) valid for $N$ uniform vortex patches as long as they satisfy the following:

(1) The maximum diameter of any patch is much smaller than the minimum distance between any two patch centroids;

(2) The centroid of any patch is within the patch itself.

The logarithmic integrands in the stream function are expanded in an infinite series about the centroids of the respective patches. This leads to an infinite system of first order ODEs in the patch centroids, measured with respect to a fixed frame, and the local geometric moments of all orders. The local geometric moment of order $m+n(m, n=0,1,2, \ldots)$ for a patch is defined as

$$
J^{(m, n)} \equiv \int_{A} \xi^{m} \eta^{n} d A
$$

where $\xi$ and $\eta$ are local coordinates in a moving frame (attached to each patch) whose origin is at the centroid of the patch, as shown in Fig. 1. On the basis of criterion 1, a small parameter can then be introduced: $\epsilon \equiv$ maximum patch diameter/minimum intercentroid distance. Defining the initial minimum intercentroid distance as a characteristic unit length it can then be seen that

$$
J^{(m, n)}=O\left(\epsilon^{m+n+2}\right) .
$$

The higher order moments are thus viewed as higher order perturbation terms. The system of ODEs is hierarchical in nature and one can obtain a closed $k$ th-order truncated model accurate to $O\left(\epsilon^{k+2}\right)$ by omitting moments of order greater than $k$ for any $k=1,2, \ldots$. Note that the zeroth order geometric moment $J^{(0,0)}$ is just the area of the patch which, as a consequence of Kelvin's circulation theorem, is conserved.

For the first order model all the moments of order greater than one are omitted. The local geometric moments of order one represent the centroid of the patch in local coordinates. These are 

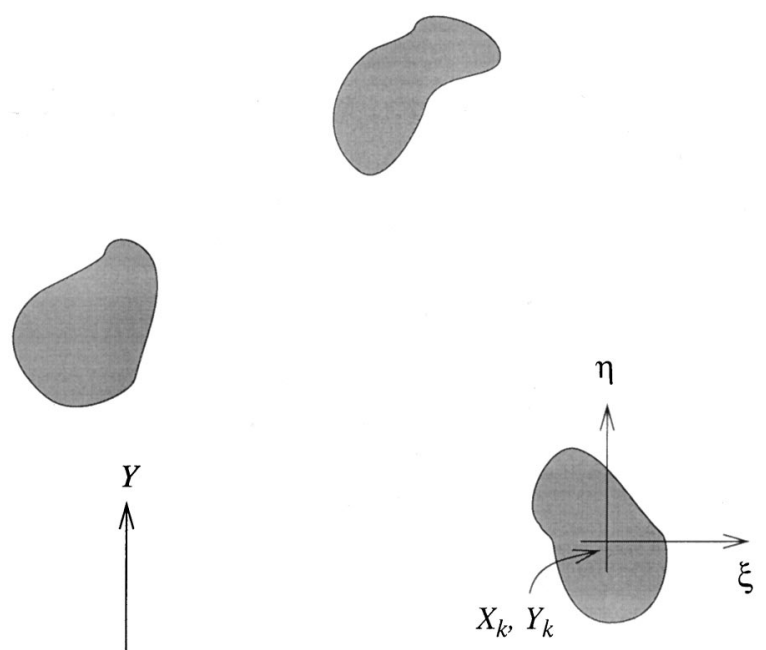

FIG. 1. A schematic representation of well-separated vortex patches in the MZS model. The local geometric moments for each patch are measured with respect to a moving frame $\xi-\eta$ fixed to the centroid of the patch. $X_{k}, Y_{k}$ are the coordinates of the centroid of the $k$ th patch in a fixed $X-Y$ frame.

zero by the choice of the local coordinate system. The truncated equations then show that the patch centroids move like a system of $N$ point vortices, hence patches at this order are approximated as point vortices.

The second order model treats each patch as a uniformly rotating Kirchhoff elliptical vortex perturbed by the presence of the other patches. The perturbation keeps the patch elliptical but changes its aspect ratio and rotation rate in general. The variables $\lambda:=$ aspect ratio and $\theta:=$ tilt of major axis, are introduced in place of the second order moments. The second order model can then be written as a closed system of ODEs in the four variables $X, Y, \lambda$, and $\theta$, where $X, Y$ are the coordinates of the centroid of the patch. As shown in Ref. 15, the following symplectic structure:

$$
\sum_{k=1}^{N} \Gamma_{k} d X_{k} \wedge d Y_{k}+\sum_{k=1}^{N} \frac{\Gamma_{k} A_{k}}{8 \pi} \frac{\lambda_{k}^{2}-1}{\lambda_{k}^{2}} d \lambda_{k} \wedge d \theta_{k}
$$

makes the system of $N$ patches a Hamiltonian system with the Hamiltonian function given by

$$
\begin{aligned}
H= & \sum_{k=1}^{N} \frac{\Gamma_{k}^{2}}{8 \pi} \log \left[\frac{\left(1+\lambda_{k}\right)^{2}}{4 \lambda_{k}}\right]+\sum_{k=1}^{N} \sum_{k<l}^{N} \frac{\Gamma_{k} \Gamma_{l}}{2 \pi} \log \hat{D}_{k l} \\
& +\sum_{k=1}^{N} \sum_{k<l}^{N} \frac{\Gamma_{k} \Gamma_{l}}{16 \pi^{2} \hat{D}_{k l}^{2}}\left[A_{l} \frac{\left(1-\lambda_{l}^{2}\right)}{\lambda_{l}} \cos \left(2\left(\phi_{k l}-\theta_{l}\right)\right)+A_{k} \frac{\left(1-\lambda_{k}^{2}\right)}{\lambda_{k}} \cos \left(2\left(\phi_{k l}-\theta_{k}\right)\right)\right],
\end{aligned}
$$

where

$$
X_{k}-X_{l}=\hat{D}_{k l} \cos \phi_{k l}, \quad Y_{k}-Y_{l}=\hat{D}_{k l} \sin \phi_{k l},
$$

$\hat{D}_{k l}$ being the distance between the centroids of patch $k$ and $l$, and $\phi_{k l}$ the angle with the reference axis made by the line joining these centroids. The Hamiltonian vector field is

$$
\frac{d X_{k}}{d t}=-\frac{1}{\Gamma_{k}} \frac{\partial H}{\partial Y_{k}}
$$




$$
\begin{gathered}
\frac{d Y_{k}}{d t}=\frac{1}{\Gamma_{k}} \frac{\partial H}{\partial X_{k}}, \\
\frac{d \lambda_{k}}{d t}=\frac{8 \pi}{\Gamma_{k} A_{k}} \frac{\lambda_{k}^{2}}{1-\lambda_{k}^{2}} \frac{\partial H}{\partial \theta_{k}}, \\
\frac{d \theta_{k}}{d t}=-\frac{8 \pi}{\Gamma_{k} A_{k}} \frac{\lambda_{k}^{2}}{1-\lambda_{k}^{2}} \frac{\partial H}{\partial \lambda_{k}} \quad k=1, \ldots, N .
\end{gathered}
$$

The phase space of the system is $P=\left(\mathbf{R}^{2}\right)^{N} \backslash C \times(U)^{N} \times\left(S^{1}\right)^{N}$, where $C:=\left\{X_{i}, Y_{i}, i\right.$ $\left.=1, \ldots, N \mid X_{j}=X_{k}, Y_{j}=Y_{k}, j \neq k\right\}$ and $U \in \mathbf{R}$ is the open interval $(1, \infty)$. The Hamiltonian is invariant under the $\mathrm{SE}(2)$ action on $P$ given by

$$
\Phi_{g}\left(X_{k}, Y_{k}, \lambda_{k}, \theta_{k}\right)=\left(X_{k} \cos \alpha-Y_{k} \sin \alpha+a_{x}, X_{k} \sin \alpha+Y_{k} \cos \alpha+a_{y}, \lambda_{k}, \theta_{k}+\alpha\right),
$$

an element $g \in \mathrm{SE}(2)$ being identified with (A,a), where $\mathbf{A} \in \mathrm{SO}(2)$ is identified with the angle $\alpha$ and $\mathbf{a} \equiv\left(a_{x}, a_{y}\right) \in \mathbf{R}^{2}$. The action is free and proper. The invariants are the components of the momentum map ${ }^{21}$ of the action, $\mathbf{J}: P \rightarrow \operatorname{se}(2)^{*}$, which is computed as

$$
\langle\mathbf{J}(p), \xi\rangle=\left\langle\left(\sum_{k=1}^{N} \Gamma_{k} \frac{X_{k}^{2}+Y_{k}^{2}}{2}+\frac{\Gamma_{k} A_{k}}{8 \pi} \frac{\lambda_{k}^{2}+1}{\lambda_{k}},-\sum_{k=1}^{N} \Gamma_{k} Y_{k}, \sum_{k=1}^{N} \Gamma_{k} X_{k}\right), \xi\right\rangle,
$$

where $p \in P, \xi \in \operatorname{se}(2)$ is an element of the Lie algebra of $\operatorname{SE}(2)$ and $\operatorname{se}(2)^{*}$ is the dual of the Lie algebra. The Lie algebra is identified with $\mathbf{R}^{3}$ and the pairing $\langle$,$\rangle on the right above is then the$ Euclidean inner product on $\mathbf{R}^{3}$. By Noether's theorem, $\mathbf{J}$ is a conserved quantity. Note the extra term appearing in the first component of $\mathbf{J}$ compared with the momentum map of $N$-point vortex motion in the unbounded plane. ${ }^{28}$ As in the point vortex problem the momentum map is equivariant iff $\sum_{k=1}^{N} \Gamma_{k}=0$. The one-cocycle for the group action which is a map $\sigma: G \rightarrow L(\mathbf{g}, C(P))$ from the group to the linear space of linear maps from the Lie algebra to the space of Casimir functions on $P$ is defined as ${ }^{21}$

$$
(\sigma(g) \cdot \xi)(p)=\left\langle\mathbf{J}\left(\Phi_{g}(p)\right), \xi\right\rangle-\left\langle\operatorname{Ad}_{g^{-1}}^{*} \mathbf{J}(p), \xi\right\rangle, \quad p \in P, \quad g \in G, \quad \xi \in \mathbf{g} .
$$

Since Casimirs are real-valued, the one-cocycle can be viewed as a map $\sigma: G \rightarrow \mathbf{g}^{*}$, for $p$ fixed. Computation of $\mathrm{Ad}_{g^{-1}}^{*}$ the coadjoint action of $\mathrm{SE}(2)$ on $\operatorname{se}(2)^{*}$ (see Appendix) gives the following one-cocycle:

$$
(\sigma(g) . \xi)(p)=\left\langle\left(\Gamma\left\{\frac{a_{x}^{2}+a_{y}^{2}}{2}\right\},-\Gamma a_{y}, \Gamma a_{x}\right), \xi\right\rangle,
$$

where $\Gamma=\Sigma_{k=1}^{N} \Gamma_{k}$. The cocycle for the $\operatorname{SE}(2)$ action in the planar $N$ point vortex problem has the same components.

The SE(2) invariance of the Hamiltonian function (4) suggests the possibility of geometric phases arising as $\mathrm{SE}(2)$ drifts. Calculation of the isotropy subgroups of the coadjoint action of $\mathrm{SE}(2)$ on $\mathrm{se}(2)^{*}$ gives an indication of the type of drifts possible in this model. The coadjoint isotropy subgroups $G_{\mu}$, where $\mu$ is a value of an $A d^{*}$-equivariant momentum map, are defined as $^{21}$

$$
G_{\mu}=\left\{g \in G \mid \operatorname{Ad}_{g-1}^{*}(\mu)=\mu\right\} .
$$

The coadjoint action of $\mathrm{SE}(2)$ (see Appendix) is given by

$$
\left\langle\operatorname{Ad}_{(A, a)^{-1}}^{*}(\eta, w),(\epsilon, v)\right\rangle=\left(\eta+\left\langle A R_{-\pi / 2} w, a\right\rangle, A w\right) .
$$


Here $\hat{\epsilon}$ is an element of the Lie algebra of $\mathrm{SO}(2)$, the vector space of $2 \times 2$ skew-symmetric (real) matrices which is identified with $\mathbf{R}$ via the isomorphism,

$$
\hat{\epsilon}=\epsilon\left(\begin{array}{cc}
0 & -1 \\
1 & 0
\end{array}\right), \quad \epsilon \in \mathbf{R}
$$

$v$ is an element of the Lie algebra of $\mathbf{R}^{2}$ which is $\mathbf{R}^{2}$ itself, and $(\eta, w) \in \operatorname{se}(2)^{*}$. For $(\eta, w)$ $=\mu$, a value of the momentum map, we get $(A, a) \in G_{\mu}$ iff

$$
\left(\eta+\left\langle A R_{-\pi / 2} w, a\right\rangle, A w\right)=(\eta, w) .
$$

This implies that

$$
\begin{aligned}
G_{\mu} & =\left\{(A, a) \in \operatorname{SE}(2) \mid A=I,\left\langle R_{-\pi / 2} w, a\right\rangle=0\right\} \simeq \mathbf{R}, \quad w \neq 0, \\
& =\operatorname{SE}(2), \quad w=0 .
\end{aligned}
$$

The condition $\left\langle R_{-\pi / 2} w, a\right\rangle=0$ picks out those elements $a \in \mathbf{R}^{2}$ parallel to $w=\left(-\sum_{k=1}^{N} \Gamma_{k} Y_{k}, \sum_{k=1}^{N} \Gamma_{k} X_{k}\right)$.

When the momentum map is not $A d^{*}$-equivariant, as in the model of two corotating patches, the coadjoint action is adjusted by the cocycle to define a new action of $G$ on $\mathbf{g}^{*}$ with respect to which the momentum map is equivariant, ${ }^{21}$

$$
g \cdot \mu=\operatorname{Ad}_{g-1}^{*}(\mu)+\sigma(g), \quad g \in G, \quad \mu \in \mathbf{g}^{*} .
$$

The isotropy subgroups with respect to this action are then defined as

$$
\widetilde{G}_{\mu}=\{g \in G \mid g \cdot \mu=\mu\} .
$$

Hence $(A, a) \in \widetilde{G}_{\mu}$ iff

$$
\left(\eta+\left\langle A R_{-\pi / 2} w, a\right\rangle, A w\right)+\left(\Gamma \frac{\|a\|^{2}}{2}, \Gamma R_{\pi / 2} a\right)=(\eta, w) .
$$

This gives two conditions that the isotropy elements have to satisfy,

$$
\begin{gathered}
\eta+\left\langle A R_{-\pi / 2} w, a\right\rangle+\Gamma \frac{\|a\|^{2}}{2}=\eta, \\
A w+\Gamma R_{\pi / 2} a=w .
\end{gathered}
$$

The second equation solved for $a$ gives $a=\left(R_{-\pi / 2}(I-A) w\right) / \Gamma$, where $I$ is the $2 \times 2$ identity matrix. This expression for $a$ identically solves the first equation since

$$
\begin{aligned}
\Gamma\left\langle A R_{-\pi / 2} w, a\right\rangle+\Gamma^{2} \frac{\|a\|^{2}}{2} & =\Gamma\left\langle A R_{-\pi / 2} w,\left(R_{-\pi / 2}(I-A) w\right) / \Gamma\right\rangle+\Gamma^{2} \frac{\|a\|^{2}}{2}, \\
& =\left\langle R_{-\pi / 2} A R_{\pi / 2} w,(I-A) w\right\rangle+\frac{1}{2}\|(I-A) w\|^{2}, \\
& =\langle A w,(I-A) w\rangle+\frac{1}{2}\left(2\|w\|^{2}-2\langle w, A w\rangle\right), \\
& =\langle A w, w\rangle-\langle A w, A w\rangle+\|w\|^{2}-\langle w, A w\rangle, \\
& =0 .
\end{aligned}
$$

Hence, 


$$
\begin{aligned}
\widetilde{G}_{\mu} & =\left\{(A, a(A)) \in \mathrm{SE}(2) \mid A \in \mathrm{SO}(2), a=\left(R_{-\pi / 2}(I-A) w\right) / \Gamma\right\}, \quad w \neq 0, \\
& =\mathrm{SO}(2), \quad w=0 .
\end{aligned}
$$

By definition the isotropy groups $G_{\mu}$ and $\widetilde{G}_{\mu}$ act on the $\mu$-level set of the momentum map, which is an invariant surface for phase space orbits. The actions are free and proper, as can be easily checked, and thus the fiber bundles $\pi_{\mu}: \mathbf{J}^{-1}(\mu) \rightarrow \mathbf{J}^{-1}(\mu) / G_{\mu}$ or $\pi_{\mu}: \mathbf{J}^{-1}(\mu)$ $\rightarrow \mathbf{J}^{-1}(\mu) / \widetilde{G}_{\mu}$ are well-defined. The total phases are elements of these groups. They measure the drifts in phase space orbits for periodic evolutions on the reduced spaces. Note that we do not investigate the existence of periodic orbits on the reduced spaces. The above geometric construction still remains valid in the absence of periodic orbits and can be used for other purposes such as study of relative equilibria in the model. The above calculations show that in the second-order MZS model these drifts can be of four types which are summarized below:

Type I: A rotation or/and a translation of the initial patch configuration if $\sum_{k=1}^{N} \Gamma_{k}=0$ and $\left(-\sum_{k=1}^{N} \Gamma_{k} Y_{k}, \sum_{k=1}^{N} \Gamma_{k} X_{k}\right)=0$.

Type II: A pure translation of the initial patch configuration if $\sum_{k=1}^{N} \Gamma_{k}=0$ and $\left(-\sum_{k=1}^{N} \Gamma_{k} Y_{k}, \sum_{k=1}^{N} \Gamma_{k} X_{k}\right) \neq 0$. The direction of translation is always parallel to the center of vorticity vector of the patch centroids.

Type III: A pure rotation of the initial patch configuration if $\sum_{k=1}^{N} \Gamma_{k} \neq 0$ and $\left(-\sum_{k=1}^{N} \Gamma_{k} Y_{k}, \sum_{k=1}^{N} \Gamma_{k} X_{k}\right)=0$.

Type IV: A rotation and a translation of the initial patch configuration if $\Sigma_{k=1}^{N} \Gamma_{k} \neq 0$ and $\left(-\sum_{k=1}^{N} \Gamma_{k} Y_{k}, \Sigma_{k=1}^{N} \Gamma_{k} X_{k}\right) \neq 0$, the translation being determined uniquely by the rotation.

To show the existense of and compute geometric phases, one has to find a connection on these bundles. The geometric phase is then computed by horizontally lifting the closed loop on the reduced space, the horizontal lift operator being defined by the connection. We do not attempt to do this in this paper. The phase space of this model does not have a canonical identification as the cotangent bundle $T^{*} Q$ of a configuration space $Q$. Hence, it is not clear if the method of inducing a connection on a cotangent bundle from a connection on the shape space bundle, $\pi: Q \rightarrow Q / G$, commonly used in simple mechanical systems, can be used here. Alternative strategies, suggested by the theory of symplectic reduction by stages for semidirect products as outlined by Marsden, Misiolek, Perlmutter, and Ratiu, ${ }^{29}$ or that of abstract mechanical connections described by Blaom ${ }^{30}$ seem more promising. The Blaom theory also helps one compute dynamic phases. However it should be noted that both these theories have been formulated for $A d^{*}$-equivariant momentum maps. To be applied to the model for two corotating patches, these theories have to be extended to admit momentum maps that are not $A d^{*}$-equivariant. A more thorough investigation (with Perlmutter and Marsden) of these and other issues is currently under way using the model of the planar $N$ point vortex problem which shares many features of this model.

\section{ADIABATIC PHASES FOR TWO COROTATING PATCHES}

The geometric phase can be more easily computed in an adiabatic setting in the second order MZS model for two corotating patches as shown in Fig. 2. The adiabatic setting can be physically described as follows. Each patch is a phase object while the background field is provided by the other patch. The relevant phase is the orientation of the patch as measured by the inclination of the major axis of the ellipse with respect to the horizontal axis. As in the point vortex problems the small parameter $\epsilon$ in the MZS model defines a ratio of two time periods or frequencies in the following way. As $\epsilon$ goes to zero the size of the patches goes to zero with respect to the intercentroid distance. The rotation and deformation of each patch would then be more influenced by its own velocity field than by the background field. We would thus expect the patch motion to approach the uniformly rotating, nondeforming motion of the Kirchhoff ellipse. If we assume that the strength of each patch and its initial aspect ratio is unaltered as $\epsilon$ varies, we can then, from (2), associate a time period $T_{s} \sim A$ with each patch. This means, in particular, that if $\epsilon$ is decreased by decreasing the area of the patches then the patches rotate increasingly fast. Simultaneously, we 


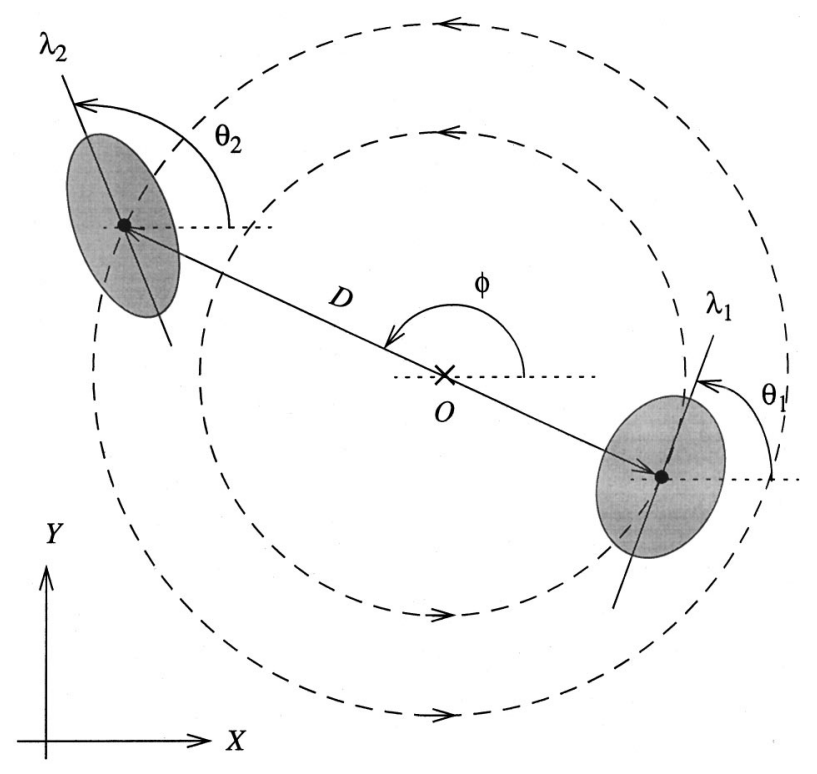

FIG. 2. The motion of two well-separated uniform elliptical vortex patches of the same sign in the MZS model. The patches have areas $A_{1}, A_{2}$ and like- signed strengths $\Gamma_{1}, \Gamma_{2}$. The motion of the centroids of the patches (small, filled circles) is, to leading order, the same as that of two point vortices of strengths $\Gamma_{1}, \Gamma_{2}$. This motion, shown by the concentric dashed circles, is about the center of vorticity of the point vortices marked $O$.

would expect the centroid motion to approach point vortex motion as discussed in the previous section. There is also a longer time period $T_{l} \sim D^{2}$, where $D$ is the typical distance between the centroids. Assuming for simplicity that $A_{1} / A_{2}=$ constant for all $\epsilon$, we define the small parameter explicitly as

$$
\epsilon^{2}=\frac{A_{1}+A_{2}}{D_{i}^{2}}
$$

where $D_{i}$ is the initial intercentroid distance. We then see that $\epsilon^{2} \sim T_{s} / T_{l}$. Therefore small values of $\epsilon$ define an adiabatic process just as in the point vortex problems in which there exist two time scales, one associated with the rotation of the patch and the other with the (relatively) slow revolution of the centroids. Using scaling arguments as in Ref. 22, one would expect that, as $\epsilon$ $\rightarrow 0$, there is an $O(1)$ contribution to the angle change of the patches at the end of the time period $T_{l}$.

The vector field considered is

$$
\begin{gathered}
\frac{d \hat{D}}{d t}=\left(\frac{\Gamma_{1}+\Gamma_{2}}{8 \pi^{2} \hat{D}^{3}}\right) \sum_{k=1}^{2} A_{k} \frac{1-\lambda_{k}^{2}}{\lambda_{k}} \sin \left(2\left(\phi-\theta_{k}\right)\right), \\
\frac{d \phi}{d t}=\frac{\Gamma_{1}+\Gamma_{2}}{2 \pi \hat{D}^{2}}-\left(\frac{\Gamma_{1}+\Gamma_{2}}{8 \pi^{2} \hat{D}^{4}}\right) \sum_{k=1}^{2} A_{k} \frac{1-\lambda_{k}^{2}}{\lambda_{k}} \cos \left(2\left(\phi-\theta_{k}\right)\right), \\
\frac{d \lambda_{1}}{d t}=\lambda_{1} \frac{\Gamma_{2}}{\pi \hat{D}^{2}} \sin \left(2\left(\phi-\theta_{1}\right)\right),
\end{gathered}
$$




$$
\begin{gathered}
\frac{d \theta_{1}}{d t}=\frac{\omega_{1} \lambda_{1}}{\left(1+\lambda_{1}\right)^{2}}+\left(\frac{1+\lambda_{1}^{2}}{1-\lambda_{1}^{2}}\right) \frac{\Gamma_{2}}{2 \pi \hat{D}^{2}} \cos \left(2\left(\phi-\theta_{1}\right)\right), \\
\frac{d \lambda_{2}}{d t}=\lambda_{2} \frac{\Gamma_{1}}{\pi \hat{D}^{2}} \sin \left(2\left(\phi-\theta_{2}\right)\right), \\
\frac{d \theta_{2}}{d t}=\frac{\omega_{2} \lambda_{2}}{\left(1+\lambda_{2}\right)^{2}}+\left(\frac{1+\lambda_{1}^{2}}{1-\lambda_{1}^{2}}\right) \frac{\Gamma_{1}}{2 \pi \hat{D}^{2}} \cos \left(2\left(\phi-\theta_{2}\right)\right) .
\end{gathered}
$$

By inspection it can be verified that this is a Hamiltonian vector field with respect to the symplectic structure,

$$
\Omega_{T}=\left(\frac{1}{\Gamma_{1}}+\frac{1}{\Gamma_{2}}\right)^{-1} \hat{D} d \hat{D} \wedge d \phi+\sum_{k=1}^{2} \frac{\Gamma_{k} A_{k}}{8 \pi} \frac{\left(\lambda_{k}^{2}-1\right)}{\lambda_{k}^{2}} d \lambda_{k} \wedge d \theta_{k}
$$

and Hamiltonian function,

$$
h=\sum_{k=1}^{2} \frac{\Gamma_{k}^{2}}{8 \pi} \log \left[\frac{\left(1+\lambda_{k}\right)^{2}}{4 \lambda_{k}}\right]+\frac{\Gamma_{1} \Gamma_{2}}{2 \pi} \log \hat{D}+\frac{\Gamma_{1} \Gamma_{2}}{16 \pi^{2} \hat{D}^{2}} \sum_{k=1}^{2} A_{k} \frac{\left(1-\lambda_{k}^{2}\right)}{\lambda_{k}} \cos \left(2\left(\phi-\theta_{k}\right)\right) .
$$

We conjecture that this is the symplectic structure residing on the reduced manifold $\mathbf{J}_{T}^{-1}(w) / \widetilde{G}_{w}$, where $\mathbf{J}_{T}$ denotes the momentum map for the action of $\mathbf{R}^{2}$ the translation subgroup of $\operatorname{SE}(2)$, and $w$ belongs to the respective Lie algebra dual. Computation of the isotropy subgroup $\widetilde{G}_{w}$ in this nonequivariant case shows that it is trivial, i.e., it consists of only the zero element. Hence, as per the Marsden-Weinstein theory, $\mathbf{J}_{T}^{-1}(w)$ is symplectic with a symplectic form defined by $\Omega_{T}$ $=i_{w}^{*} \Omega$, where $i_{w}: \mathbf{J}_{T}^{-1}(w) \rightarrow P$ is the inclusion map and $\Omega$ is given by (3).

We make the following additional remarks about this system of equations:

(1) The equations clearly show that for large $\hat{D}$ or small $A_{1}, A_{2}$ the centroids move like two point vortices of strengths $\Gamma_{1}$ and $\Gamma_{2}$ respectively;

(2) The $\lambda_{k}, \theta_{k}$ variables represent the patch deformation and rotation, respectively. Their equations are the same as Eqs. (3.19) and (3.20) in Ref. 15. They show that for large $\hat{D}$ or small $A_{1}, A_{2}$ (equivalently large $\omega_{1}, \omega_{2}$ ) the patches rotate like Kirchhoff ellipses.

(3) The $\lambda_{k}, \theta_{k}$ equations for a patch are not explicitly dependent on the $\lambda_{k}, \theta_{k}$ of the other patch. The mutual interdependency comes only through the $\hat{D}$ and $\phi$ variables. However, there is a direct dependency on the strength of the other patch;

(4) As pointed out in Ref. 15 the $\theta_{1}, \theta_{2}$ equations are not defined for $\lambda_{k}=1$, i.e., circular patches.

We now proceed to nondimensionalize these equations and calculate the geometric phase in the angle variables $\theta_{1}$ and $\theta_{2}$ at the end of one long time period $T_{l}$ of the centroid motion. The phase appears as an additional $O(1)$ term in the leading order change in these variables. The method of computation is the standard two time scale asymptotic procedure described in Refs. 22 and 25. The details of the calculations are not given in this paper but may be found in Ref. 31, only the final results are stated. The geometric interpretation is made in the next subsection.

Introduce the nondimensional variables,

$$
D=\frac{\hat{D}}{D_{i}}, \quad t=\Omega \hat{t}
$$


where $\Omega=\left(\Gamma_{1}+\Gamma_{2}\right) /\left(A_{1}+A_{2}\right) . \Omega$ is the mean strength over the mean area of the patches and represents the time scale of the "unperturbed" frequency of the patches. This gives the following equations in the nondimensional variables:

$$
\begin{gathered}
\frac{d D}{d t}=\left(\frac{\epsilon^{4}}{8 \pi^{2} D^{3}}\right) \sum_{k=1}^{2} \sigma_{k} f\left(\lambda_{k}\right) \sin \left(2\left(\phi-\theta_{k}\right)\right), \\
\frac{d \phi}{d t}=\left(\frac{\epsilon^{2}}{2 \pi D^{2}}\right)-\left(\frac{\epsilon^{4}}{8 \pi^{2} D^{4}}\right) \sum_{k=1}^{2} \sigma_{k} f\left(\lambda_{k}\right) \cos \left(2\left(\phi-\theta_{k}\right)\right), \\
\frac{d \lambda_{1}}{d t}=\epsilon^{2} \lambda_{1} \frac{\alpha_{2}}{\pi D^{2}} \sin \left(2\left(\phi-\theta_{1}\right)\right), \\
\frac{d \theta_{1}}{d t}=\frac{\alpha_{1} g\left(\lambda_{1}\right)}{\bar{A}_{1}}+\epsilon^{2} \frac{\alpha_{2} h\left(\lambda_{1}\right)}{2 \pi D^{2}} \cos \left(2\left(\phi-\theta_{1}\right)\right), \\
\frac{d \lambda_{2}}{d t}=\epsilon^{2} \lambda_{2} \frac{\alpha_{1}}{\pi D^{2}} \sin \left(2\left(\phi-\theta_{2}\right)\right), \\
\frac{d \theta_{2}}{d t}=\frac{\alpha_{2} g\left(\lambda_{2}\right)}{\bar{A}_{2}}+\epsilon^{2} \frac{\alpha_{1} h\left(\lambda_{2}\right)}{2 \pi D^{2}} \cos \left(2\left(\phi-\theta_{2}\right)\right),
\end{gathered}
$$

where $\sigma_{k}=A_{k} /\left(A_{1}+A_{2}\right), \alpha_{k}=\Gamma_{k} /\left(\Gamma_{1}+\Gamma_{2}\right), f(\lambda)=\left(1-\lambda^{2}\right) / \lambda, g(\lambda)=\lambda /(1+\lambda)^{2}$, and $h(\lambda)$ $=\left(1+\lambda^{2}\right) /\left(1-\lambda^{2}\right)$. The initial conditions for this system are

$$
D(0)=1, \phi(0), \theta_{1}(0), \theta_{2}(0), \lambda_{1}(0), \lambda_{2}(0) .
$$

Choosing $\tau=\epsilon^{2} t$ as the slow time scale and performing the multiscale calculation gives the following asymptotic results. The solutions to leading order are

$$
\begin{gathered}
D=1+O(\epsilon), \\
\phi=\frac{\tau}{2 \pi}+\phi(0)+O(\epsilon), \\
\lambda_{k}=\lambda_{k}(0)+O(\epsilon), \\
\theta_{k}=\frac{\alpha_{k}}{\sigma_{k}} g\left(\lambda_{k}(0)\right) t-\frac{\alpha_{k^{\prime}}}{2 \pi} \frac{1-\lambda_{k}(0)}{1+\lambda_{k}(0)} \cos \left(2\left(\phi(0)-\theta_{k}(0)\right)\right) \cdot \tau+\theta_{k}(0)+O(\epsilon),
\end{gathered}
$$

where $k^{\prime}=2$ if $k=1$ and vice versa. The change in the patch orientation angles $\Delta \theta_{k}(k=1,2)$ at the end of the long time period, $T=4 \pi^{2}$ (to leading order), are given by

$$
\Delta \theta_{k}=\frac{\alpha_{k}}{\sigma_{k}} g\left(\lambda_{k}(0)\right) \frac{4 \pi^{2}}{\epsilon^{2}}-\alpha_{k^{\prime}} \frac{1-\lambda_{k}(0)}{1+\lambda_{k}(0)} 2 \pi \cos \left(2\left(\phi(0)-\theta_{k}(0)\right)\right)+O\left(\epsilon^{2}\right) .
$$

Thus,

Proposition: The adiabatic geometric phase for a system of two elliptical corotating vortex patches evolving according to the equations of the second-order truncated MZS model is given by 


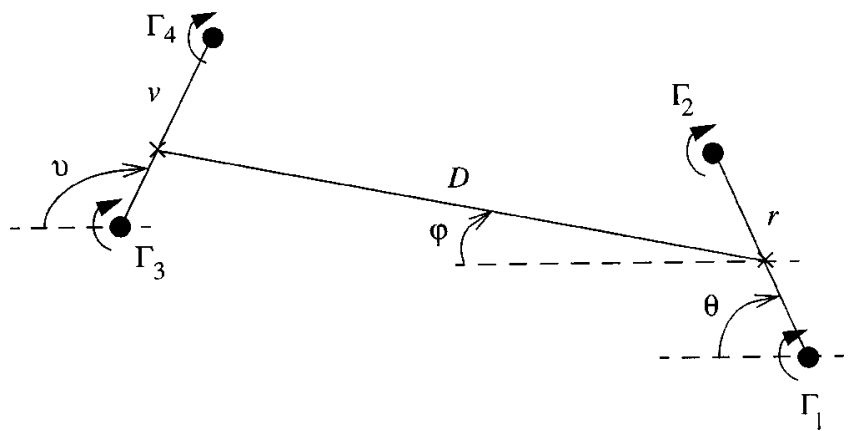

FIG. 3. Four point vortices (filled circles) of positive strengths in an unbounded plane. The geometric phase is calculated for the variables $\theta$ and $v$. The $\times \mathrm{s}$ mark the centers of vorticity of each pair, respectively.

$$
\begin{aligned}
& \left(\theta_{g}\right)_{1}=-\left(\frac{\Gamma_{2}}{\Gamma_{1}+\Gamma_{2}}\right)\left(\frac{1-\lambda_{1}(0)}{1+\lambda_{1}(0)}\right) 2 \pi \cos \left(2\left(\phi(0)-\theta_{1}(0)\right)\right) \\
& \left(\theta_{g}\right)_{2}=-\left(\frac{\Gamma_{1}}{\Gamma_{1}+\Gamma_{2}}\right)\left(\frac{1-\lambda_{2}(0)}{1+\lambda_{2}(0)}\right) 2 \pi \cos \left(2\left(\phi(0)-\theta_{2}(0)\right)\right)
\end{aligned}
$$

where the $\Gamma s$ and $\lambda s$ refer to the strengths and the aspect ratios of the patches, respectively.

To compare with an analogous point-vortex problem, we present the results of similar phase computations of the four-vortex configuration shown in Fig. 3. There are now, in the terminology of Ref. 22, two phase object-parent vortex pairs with each pair playing the role of farfield vortices for the other pair. When these two pairs are well separated we expect their slow motion to approach that of two vortices of strengths $\Gamma_{1}+\Gamma_{2}$ and $\Gamma_{3}+\Gamma_{4}$, respectively. We therefore have an asymptotically defined closed orbit as in the three-vortex problem and we try to see if each pair experiences an adiabatic geometric phase at the end of one period of this two-vortex motion. The geometric phase obtained in $\theta$ and $\nu$ is

$$
\begin{aligned}
\theta_{g} & =\frac{\Gamma_{3}+\Gamma_{4}}{\Gamma_{1}+\Gamma_{2}+\Gamma_{3}+\Gamma_{4}} 2 \pi \cos 2 \theta_{i}, \\
\nu_{g} & =\frac{\Gamma_{1}+\Gamma_{2}}{\Gamma_{1}+\Gamma_{2}+\Gamma_{3}+\Gamma_{4}} 2 \pi \cos 2 \nu_{i} .
\end{aligned}
$$

For $\Gamma_{4}=0, \theta_{g}$ gives the geometric phase for the three-vortex problem derived in Ref. 22 and $\nu_{g}$ gives the geometric phase for a passive particle in the vicinity of $\Gamma_{3}$ in the three-vortex problem.

Comparing with the results of the patch problem, we find that the geometric phase for each of the two vortex patches is the geometric phase of a corresponding pair of point vortices in this four-vortex configuration, where the sum of the strengths of the two vortices in a pair is the patch strength, times the factor $\left(1-\lambda_{k}(0)\right) /\left(1+\lambda_{k}(0)\right)(k=1,2)$. This factor can be viewed as the contribution to the geometric phase of the internal structure associated with each patch.

We point out here that the results for both the patch and the point vortex problem clearly depend upon the initial conditions, unlike the adiabatic phases in the works of Hannay and Berry ${ }^{16,17}$ In particular, to make direct contact with this work, one would average the initial orientations $\phi(0)-\theta_{i}(0)$ over the full circle and obtain zero for these averaged phases. In the context of point vortex models, we have shown ${ }^{23}$ that these phases, despite averaging to zero, are 
important when computing quantities in the flow, such as interface stretching rates. We expect the same to be true when one replaces point vortices with vortex patches.

\section{GEOMETRIC INTERPRETATION}

As shown by Simon ${ }^{18}$ and Marsden, Montgomery, and Ratiu, ${ }^{19}$ the process of averaging in parameter-dependent Hamiltonian defines a connection on the trivial principal fiber bundle $\pi: M$ $\times P \rightarrow P$, where $P$ is the parameter space and $M$, for an $n$-dimensional integrable Hamiltonian, is the level set of the $n$ independent integrals of the system with the parameters frozen. For $M$ compact and connected this set is diffeomorphic to the $n$-torus. Viewing the patch system as a "parameter"-dependent Hamiltonian system with the patch centroids as the "parameters," the choice of the following fiber bundle for defining an adiabatic connection seems natural. Define $E=\pi: T^{2} \times S^{1} \rightarrow S^{1}$, where $T^{2}=S^{1} \times S^{1}$ is the 2-torus. $T^{2}$ is identified with $M$. As the adiabatic limit is approached the patch centroids are frozen, relative to the patch motion, and each patch behaves locally in time like a Kirchhoff ellipse. For a Kirchhoff patch, $M=S^{1}$ the level set of the momentum map (8). For two patches we then define $M$ as the product of the level set for each patch, i.e., $M=T^{2}$. The patch centroids evolve locally in time like point vortices of the same strength, and therefore the "parameter" space for two patches is chosen as the level set of the momentum map [of the $\mathrm{SE}(2)$ action] in the two point vortex problem. All these level sets are diffeomorphic to $S^{1}$ (for like-signed vortices), and this is our choice for our base space in $E$.

The next step is to show that the leading order motion of the patches defines a connection on this trivial principal fiber bundle. The leading order motion of each patch was obtained in Sec. III as (dropping the subscript $k$ )

$$
\begin{gathered}
\lambda^{(0)}=\text { constant, } \\
\theta^{(0)}=\theta_{F}(t)+\theta_{S}(\tau) .
\end{gathered}
$$

An examination of this relation reveals a connection. Indeed $\theta_{S}(\tau)$ defines a local connection 1-form, i.e., a Lie-algebra valued differential 1-form on the base space $S^{1}$ of the bundle $E$ in the following manner. The nondimensional slow time varaiable $\tau$ can be viewed as a coordinate for the one-dimensional base space $S^{1}$, since all the circular vortex orbits of the two-(point)vortex motion (diffeomorphic to $S^{1}$ ) depend on the slow time alone. A change of coordinates from $\tau$ to $\phi$ can be affected through the relation $\phi=\tau / 2 \pi$. Define $\omega=d \phi / d \tau$ as the common nondimensionalized angular velocity of two-(point)vortex motion. The term $\theta_{S}(\tau)$ being linear in $\tau$ one can rewrite it as

$$
\theta_{S}(\tau)=\int_{0}^{\tau} \frac{d \theta_{S}}{d \tau} d \tau=\frac{\omega_{S}}{\omega} \phi,
$$

where $\omega_{S}$ can be viewed, nominally, as the angular velocity of the slow drift that cumulatively gives the geometric phase. This defines the following equation between differentials:

$$
d \theta_{S}=\frac{\omega_{S}}{\omega} d \phi \equiv v d \phi, \quad v \in \mathbf{R} .
$$

The right-hand side of the above equation defines a 1-form on the base manifold $S^{1}$ (with coordinate $\phi$ ). Indeed, since there are two patches we get the following $\mathbf{R}^{2}$-valued 1-form on $S^{1}$ :

$$
\gamma_{*}=\left(v_{1} d \phi ; v_{2} d \phi\right), \quad v_{1}, v_{2} \in \mathbf{R}
$$

(where we have reintroduced the subscripts). In (15) and (16) the tangent space at each point of $S^{1}$ is identified with the tangent line $(\equiv \mathbf{R})$, embedded in $\mathbf{R}^{2}$, at that point. The 1 -form $d \phi$ at the point $\phi$ is then just the canonical representation of a 1-form on $\mathbf{R}^{2}$. Now consider the action of $T^{2}$ on $E$ by left translations, $\left(\alpha_{1}, \alpha_{2}, \phi\right) \rightarrow\left(\theta_{1}+\alpha_{1}, \theta_{2}+\alpha_{2}, \phi\right)$. This induces an isomorphism of the 
tangent spaces of $T^{2}$ which, by viewing the tangent spaces as two lines each embedded in a copy of $\mathbf{R}^{2}$, is a rotation of tangent vectors by $\theta_{1}$ and $\theta_{2}$, respectively. The image vectors in (16) can then be identified with Lie algebra elements and this allows the definition of a Lie-algebra valued local connection 1 -form on the base space $S^{1}$,

$$
\gamma=\left(R_{-\theta_{1}} v_{1} d \phi, R_{-\theta_{2}} v_{2} d \phi\right)
$$

where $R_{\theta_{1}}$ denotes the $2 \times 2$ matrix which rotates vectors in $\mathbf{R}^{2}$ by $\theta_{1}$. The prescription for finding a connection form on the bundle $E$ is then straightforward. One simply "adds" the trivial connection 1-form to the local connection 1-form. Thus, we have:

Theorem: The adiabatic geometric phase in the second order MZS model of corotating vortex patches can be viewed as the holonomy of a flat connection on the trivial principal bundle $\pi: E$ $=T^{2} \times S^{1} \rightarrow S^{1}$. The base space $S^{1}$ is diffeomorphic to the momentum level set of the two-pointvortex motion of the patch centroids and the fiber at each point is the product of the momentum level set of each patch. The Lie algebra valued connection form is given by

$$
A=\left(R_{-\theta_{1}} d \theta_{1}-R_{-\theta_{1}} v_{1} d \phi, R_{-\theta_{2}} d \theta_{2}-R_{-\theta_{2}} v_{2} d \phi\right)
$$

where $\left(\theta_{1}, \theta_{2}, \phi\right)$ are coordinates on $E$ and $v_{k}$ is the ratio of the constant slow phase "angular velocity," $d\left(\theta_{S}\right)_{k} / d \tau$, induced on patch $k$ by the other patch, and the angular velocity, $\omega(\phi)$, of the point vortex in the closed orbit.

Proof: Both properties of a connection form are trivially satisfied. The $T^{2}$ action on $E$ is given by fiber-invariant translations $\Phi_{g}\left(\alpha_{1}, \alpha_{2}, \phi\right)=\left(\theta_{1}+\alpha_{1}, \theta_{2}+\alpha_{2}, \phi\right)$, where $g \equiv\left(\theta_{1}, \theta_{2}\right) \in T^{2}$. The infinitesimal generator of the action is the vector field $\xi_{E}\left(\theta_{1}, \theta_{2}, \phi\right)=\left(R_{\theta_{1}} \zeta, R_{\theta_{2}} \beta, 0\right)$, where $(\zeta, \xi) \equiv \xi \in \mathbf{g}$ (the Lie algebra of $T^{2}$ ). Hence, $A\left(\xi_{E}\right)=\xi$.

Next, the adjoint action is the identity since $T^{2}$ is Abelian. The derivative of the group action is given by $d \Phi_{g}(p)\left(w_{1}, w_{2}, 0\right)=\left(R_{\theta_{1}} w_{1}, R_{\theta_{2}} w_{2}, 0\right)$, at $p \equiv\left(\alpha_{1}, \alpha_{2}, \phi\right)$ and where $\left(w_{1}, w_{2}, 0\right)$ is a vector tangent to the fiber at $p$. Thus, $A \cdot d \Phi_{g}(p)=\left(R_{-\theta_{1}-\alpha_{1}} \cdot R_{\theta_{1}} w_{1}, R_{-\theta_{2}-\alpha_{2}} \cdot R_{\theta_{2}} w_{2}\right)$ $=\left(R_{-\alpha_{1}} w_{1}, R_{-\alpha_{2}} w_{2}\right)=\operatorname{Ad}_{g} \cdot A(p) \forall g \in T^{2}$

The existense of a connection implies a direct sum decomposition $T_{p} E=H_{p} \oplus V_{p}$. Any vector $\left(\eta_{\theta_{1}}, \eta_{\theta_{2}}, \eta_{\phi}\right) \in T_{p} E$ can be written uniquely as

$$
\left(\eta_{\theta_{1}}, \eta_{\theta_{2}} \eta_{\phi}\right)=\left(\eta_{\theta_{1}}-v_{1} \eta_{\phi}, \eta_{\theta_{2}}-v_{2} \eta_{\phi}, 0\right)+\left(v_{1} \eta_{\phi}, v_{2} \eta_{\phi}, \eta_{\phi}\right)
$$

where $\left(\eta_{\theta_{1}}-v_{1} \eta_{\phi}, \eta_{\theta_{2}}-v_{2} \eta_{\phi}, 0\right) \equiv V_{p}$ is the vertical part and $\left(v_{1} \eta_{\phi}, v_{2} \eta_{\phi}, \eta_{\phi}\right) \in H_{p}$ is the horizontal part. $H_{p}$ [the span of all vectors $\left.\left(v_{1} \eta_{\phi}, v_{2} \eta_{\phi}, \eta_{\phi}\right)\right]$ is a one-dimensional subspace of $T_{p} E$ at each $p$. It is the range of the horizontal lift operator defined by $\operatorname{hor}_{p}\left(d \pi\left(v_{1} \eta_{\phi}, v_{2} \eta_{\phi}, \eta_{\phi}\right)\right)$ $=\left(v_{1} \eta_{\phi}, v_{2} \eta_{\phi}, \eta_{\phi}\right)$.

The total phase change in the patch can therefore now be interpreted in terms of the connection. The constant-frequency constant-separation motion of each unperturbed Kirchhoff patch is represented by an orbit on $E$ with coordinates $\left(\left(\theta_{F}(t)\right)_{1},\left(\theta_{F}(t)\right)_{2}, \phi=\right.$ constant $)$. The leading order perturbation term due to the other patch, is $\theta_{S}$, causing these circular orbit on $E$ to drift and thus wind around the 3-torus. The orbit is now defined by the coordinates

$$
\left\{\left(\theta_{F}(t)\right)_{1}+\left(\theta_{S}(t)\right)_{1},\left(\theta_{F}(t)\right)_{2}+\left(\theta_{S}(t)\right)_{2}, \phi(t)\right\}
$$

The tangent vector to this orbit at each $e \in E$ can be written according to (17) as 


$$
\left(\frac{d \theta_{1}}{d t}, \frac{d \theta_{2}}{d t}, \frac{d \phi}{d t}\right) \equiv\left(\frac{d \theta_{1}}{d t}-v_{1} \frac{d \phi}{d t}, \frac{d \theta_{2}}{d t}-v_{2} \frac{d \phi}{d t}, 0\right)+\left(v_{1} \frac{d \phi}{d t}, v_{2} \frac{d \phi}{d t}, \frac{d \phi}{d t}\right)
$$

Using (15) and (18), we can rewrite this as

$$
\begin{aligned}
\left(\frac{d \theta_{1}}{d t}, \frac{d \theta_{2}}{d t}, \frac{d \phi}{d t}\right) & \equiv\left(\frac{d \theta_{1}}{d t}-\frac{d\left(\theta_{S}\right)_{1}}{d t}, \frac{d \theta_{2}}{d t}-\frac{d\left(\theta_{S}\right)_{2}}{d t}, 0\right)+\left(\frac{d\left(\theta_{S}\right)_{1}}{d t}, \frac{d\left(\theta_{S}\right)_{2}}{d t}, \frac{d \phi}{d t}\right), \\
& \equiv\left(\frac{d\left(\theta_{F}\right)_{1}}{d t}, \frac{d\left(\theta_{F}\right)_{2}}{d t}, 0\right)+\left(\frac{d\left(\theta_{S}\right)_{1}}{d t}, \frac{d\left(\theta_{S}\right)_{2}}{d t}, \frac{d \phi}{d t}\right) .
\end{aligned}
$$

This clearly shows that the vertical part of the tangent vector is the rate of evolution at the unperturbed frequency leading to the dynamic phase and the vertical drift due to the horizontal part gives the geometric phase at the end of one circuit around the base circle.

The geometric interpretation of the phase in the four point vortex problem is similarly made. The fibers of the principal bundle $\pi: T^{2} \times S^{1} \rightarrow S^{1}$ are identified with the product of the momentum level set of each vortex pair and the base is diffeomorphic to the momentum level set of the two vortex motion of the pair centroids in the adiabatic limit. The connection on this bundle is then constructed exactly as before.

\section{ACKNOWLEDGMENTS}

We would like to thank Fred Browand and Jerry Marsden for bringing the MZS model to our attention. P.K.N. would like to thank J. Hannay for discussions regarding the initial condition dependence on calculations of the phase. B.N.S. would also like to thank Matthew Perlmutter for assistance in computing the coadjoint isotropy subgroups, and Richard Murray for the support of a postdoctoral fellowship. P.K.N. was partially supported by NSF-DMS-9800797.

\section{APPENDIX}

To compute the coadjoint action of $\mathrm{SE}(2)$, recall some basic facts about the $\mathrm{SE}(2)$ group. Group composition of elements is given by

$$
(A, a) \cdot(B, b)=(A B, A b+a)
$$

with the identitiy element $(I, 0)$. The inverse of an element $(A, a)$ is $\left(A^{-1},-A^{-1} a\right)$. Inner automorphisms are thus given by

$$
I_{(A, a)}(B, b):=(A, a) \cdot(B, b) \cdot(A, a)^{-1}=(B,-B a+A b+a) .
$$

The derivative of the inner automorhphism corresponding to $(A, a)$ gives the adjoint action of $(A, a)$ on $g$,

$$
\operatorname{Ad}_{(A, a)}(\hat{\boldsymbol{\epsilon}}, v)=\left.\frac{d}{d t}\right|_{t=0} I_{(A, a)}(B(t), b(t))=(\hat{\boldsymbol{\epsilon}},-\hat{\boldsymbol{\epsilon}} a+A v)
$$

where $(B(t), b(t))$ is a smooth curve in $\mathrm{SE}(2)$ with $B(0)=I, \dot{B}(0)=\hat{\epsilon}, b(0)=0, \dot{b}(0)=v$. Here $\hat{\epsilon}$ is an element of the Lie algebra of $\mathrm{SO}(2)$, the vector space of $2 \times 2$ skew-symmetric (real) matrices which is identified with $\mathbf{R}$ via the isomorphism,

$$
\hat{\epsilon}=\epsilon\left(\begin{array}{cc}
0 & -1 \\
1 & 0
\end{array}\right), \quad \epsilon \in \mathbf{R}
$$


and $v$ is an element of the Lie algebra of $\mathbf{R}^{2}$ which is $\mathbf{R}^{2}$ itself. As vector spaces we make the usual identification $\operatorname{se}(2) \equiv \mathbf{R} \times \mathbf{R}^{2} \equiv \mathbf{R}^{3}$. The coadjoint action of $(A, a)$ on $\operatorname{se}(2)^{*}$ is then computed relative to the Euclidean inner product $\langle$,$\rangle on \mathbf{R}^{3}$. It is a standard calculation and we recall the final result,

$$
\left\langle\operatorname{Ad}_{(A, a)^{-1}}^{*}(\eta, w),(\epsilon, v)\right\rangle=\left(\eta+\left\langle A R_{-\pi / 2} w, a\right\rangle, A w\right) .
$$

Here $R_{\pi / 2}$ denotes the matrix in (A1), and $R_{-\pi / 2}$ its inverse.

${ }^{1}$ H. Lamb, Hydrodynamics, 6th ed. (Dover, New York 1932).

${ }^{2}$ G. S. Deem and N. J. Zabusky, 'Vortex waves: stationary 'V states,' interactions, recurrence, and breaking," Phys. Rev. Lett. 40, 859-862 (1978).

${ }^{3}$ E. A. Overman and N. J. Zabusky, "Coaxial scattering of Euler-equation translating $V$ states via contour dynamics," J. Fluid Mech. 125, 187-202 (1982).

${ }^{4}$ E. A. Overman II and N. J. Zabusky, "Evolution and merger of isolated vortex structures,” Phys. Fluids 25, 1297-1305 (1982).

${ }^{5}$ S. Kida, "Motion of an elliptical vortex in a uniform shear flow,'” J. Phys. Soc. Jpn. 50, 3517-3520 (1981).

${ }^{6}$ Y. H. Wan and M. Pulvirente, "Nonlinear stability of circular vortex patches," Commun. Math. Phys. 99, 435-450 (1984).

${ }^{7}$ Y. H. Wan, "The stability of rotating vortex patches," Commun. Math. Phys. 107, 1-20 (1986).

${ }^{8}$ P. Constantin and E. Titi, "On the evolution of nearly circular vortex patches," Commun. Math. Phys. 119, 177-198 (1988).

${ }^{9}$ P. G. Saffman, Vortex Dynamics, Cambridge Monographs on Mechanics and Applied Mathematics (Cambridge University Press, Cambridge 1992).

${ }^{10} \mathrm{P}$. J. Morrison and J. M. Greene, "Noncanonical Hamiltonian density formulation of hydrodynamics and ideal magnetohydrodynamics," Phys. Rev. Lett. 45, 790-794 (1980); 48, 569 (1982).

${ }^{11}$ P. J. Olver, "A nonlinear Hamiltonian structure for the Euler equations," J. Math. Anal. Appl. 89, 233-250 (1982).

${ }^{12}$ J. Marsden and A. Weinstein, "Coadjoint orbits, vortices, and Clebsch variables for incompressible fluids," Physica D 7, 305-323 (1983).

${ }^{13}$ T. G. Shepherd, "Symmetries, conservation laws and Hamiltonian structure in geophysical fluid dynamics," Adv. Geophys. 32, 287-338 (1990).

${ }^{14}$ M. V. Melander, A. S. Styczek, and N. J. Zabusky, "Elliptically desingularized model for the two-dimensional Euler equations,"' Phys. Rev. Lett. 53, 1222-1225 (1984).

${ }^{15}$ M. V. Melander, N. J. Zabusky, and A. S. Styczek, “Moment model for vortex interactions. Part I,' J. Fluid Mech. 167, 95-115 (1986).

${ }^{16}$ M. V. Berry, “Quantal phase factors accompanying adiabatic changes,” Proc. R. Soc. London, Ser. A 392, 45-57 (1984).

${ }^{17}$ J. Hannay, “Angle variable holonomy in adiabatic excursion of an integrable Hamiltonian,” J. Phys. A 18, 221-230 (1985).

${ }^{18}$ B. Simon, "Holonomy, the quantum adiabatic theorem, and Berry's phase," Phys. Rev. Lett. 51, 2167-2170 (1983).

${ }^{19}$ J. E. Marsden, R. Montgomery, and T. S. Ratiu, "Reduction, symmetry, and phases in mechanics," AMS Memoirs 436 (1990).

${ }^{20} \mathrm{R}$. Montgomery, "The connection whose holonomy is the classical adiabatic angles of Hannay and Berry and its generalization to the nonintegrable case," Commun. Math. Phys. 120, 269-294 (1988).

${ }^{21}$ J. E. Marsden and T. S. Ratiu, Introduction to Mechanics and Symmetry, Texts in Applied Mathematics 17, 2nd ed. (Springer-Verlag, Berlin, 1999).

${ }^{22}$ B. N. Shashikanth and P. K. Newton, "Vortex motion and the geometric phase. Part I: Basic configurations and asymptotics," J. Nonlinear Sci. 8, 183-214 (1998).

${ }^{23}$ B. N. Shashikanth and P. K. Newton, "Vortex motion and the geometric phase. Part II: Slowly varying spiral structures," J. Nonlinear Sci. 9, 233-254 (1999).

${ }^{24}$ P. K. Newton, The N-Vortex Problem: Analytical Techniques, Applied Mathematical Sciences (Springer-Verlag, Berlin, 2001) (to be published).

${ }^{25}$ P. K. Newton, “Hannay-Berry phase and the restricted three-vortex problem," Physica D 79, 416-423 (1994).

${ }^{26}$ P. K. Newton and B. N. Shashikanth, "Vortex problems, rotating spiral structure, and the Hannay-Berry phase," Second International Workshop on Vortex Flows and Related Numerical Methods, Montreal, Canada, 1995. ESAIM Proceedings, Soc. Math. Appl. Indust., Inst. Henri Poincare, 1996.

${ }^{27}$ J. Kevorkian and J. D. Cole, Multiple Scale and Singular Perturbation Methods, Applied Mathematical Sciences (Springer-Verlag, Berlin, 1996), p. 114.

${ }^{28}$ M. Adams and T. S. Ratiu, "The three point vortex problem: Commutative and noncommutative integrability," Contemp. Math. 81, 245-257 (1988).

${ }^{29}$ J. E. Marsden, G. Misiolek, M. Perlmutter, and T. S. Ratiu, "Symplectic reduction for semidirect products and central extensions," Diff. Geom. Applic. 9, 173-212 (1998).

${ }^{30}$ A. Blaom, "Reconstruction phases via Poisson reduction," Diff. Geom. Applic. 12, 231-252 (2000).

${ }^{31}$ B. N. Shashikanth, "Vortex motion and the geometric phase," Ph.D. thesis, University of Southern California, 1997. 